PROFESSOR MONICA PENTENERO (Orcid ID : 0000-0003-3972-1203)

DR STEFANO FEDELE (Orcid ID : 0000-0001-9006-9412)

Received Date : 12 -Aug-2016

Revised Date : 04-Dec-2016

Accepted Date : 20-Dec-2016

Article type : Original Manuscript

\title{
Time to onset of bisphosphonate-related osteonecrosis of the jaws: a multicentre retrospective cohort study
}

Fung PPL, ${ }^{1}$ Bedogni G, ${ }^{2}$ Bedogni A, ${ }^{3,4}$ Petrie A, ${ }^{1}$ Porter $S,{ }^{1}$ Campisi G, ${ }^{5}$ Bagan J, ${ }^{6}$ Fusco V, ${ }^{7}$ Saia G, ${ }^{4}$ Acham S, 8 Musto P, 9 Petrucci MT, ${ }^{10}$ Diz P, ${ }^{11}$ Colella G, 12 Mignogna MD, ${ }^{13}$ Pentenero M, 14 Arduino P, ${ }^{15}$ Lodi G, 16 Maiorana C, ${ }^{17}$ Manfredi M, 18, Hallberg P, ${ }^{19}$ Wadelius M, 19 Takaoka K, 20 Leung YY, ${ }^{21}$ Bonacina R, ${ }^{22}$ Schiødt M, ${ }^{23}$ Lakatos P, ${ }^{24}$ Taylor T, 25 De Riu G, ${ }^{26}$ Favini G, ${ }^{27}$ Rogers SN, ${ }^{28}$ Pirmohamed M, ${ }^{29}$ Nicoletti, P, ${ }^{30}$ GENVABO Consortium, ${ }^{31}$ Fedele S. ${ }^{1,32}$

1. University College London / University College London Hospital Eastman Dental Institute and Hospital, London UK

2. Liver Research Centre, Clinical Epidemiology Unit, Basovizza, Trieste, Italy

3. University of Verona, Department of Maxillofacial Surgery, Verona, Italy

4. University of Padua, Department of Maxillofacial Surgery, Padua, Italy

5. University of Palermo, Dip. Discipline Chirurgiche, Oncologiche e Stomatologiche, Palermo, Italy

6. Valencia University, Oral Medicine, University General Hospital, Department of Oral and Maxillofacial Surgery, Valencia, Spain.

7. Ospedale SS Antonio e Biagio e C Arrigo, Department of Oncology and Haematology, Medical Oncology Unit, Alessandria, Italy

8. Medical University of Graz, University Clinic of Dental Health and Oral Medicine, Department of Oral Surgery and Orthodontics, Graz, Austria

9. IRCCS, Referral Cancer Center of Basilicata, Scientific Direction, Rionero in Vulture (Potenza), Italy

10. "Sapienza" University, Department of Cellular Biotechnologies and Haematology, Rome, Italy

11. Santiago de Compostela University, School of Medicine and Dentistry, Santiago de Compostela, Spain

This article has been accepted for publication and undergone full peer review but has not been through the copyediting, typesetting, pagination and proofreading process, which may lead to differences between this version and the Version of Record. Please cite this article as doi: 10.1111/odi.12632

This article is protected by copyright. All rights reserved. 
12. Second University of Naples, Department of Medical, Surgical and Dental Specialties, Naples, Italy

13. Federico II University, Department of Neurosciences, Reproductive and Odontostomatological Sciences, Head \& Neck Clinical Section, Naples, Italy

14. University of Torino, Department of Oncology, Oral Medicine and Oral Oncology Unit, Turin, Italy

15. University of Turin, CIR Dental School, Turin, Italy

16. Università degli Studi di Milano, Dipartimento di Scienze Biomediche, Chirurgiche e Odontoiatriche, Milan, Italy.

17. Fondazione IRCCS Policlinico Cà Granda, Ospedale Maggiore Policlinico, and University of Milan, Dipartimento di Scienze Biomediche, Chirurgiche ed Odontoiatriche, Italy

18. Parma University, Dipartimento di Scienze Biomediche, Biotecnologiche e Traslazionali S.Bi.Bi.T., Unità di Odontostomatologia, Parma, Italy

19. Uppsala University, Department of Medical Sciences, Clinical Pharmacology and Science for Life Laboratory, Uppsala, Sweden

20. Hyogo College of Medicine, Department of Oral and Maxillofacial Surgery, Japan

21. Oral and Maxillofacial Surgery, Faculty of Dentistry, The University of Hong Kong

22. Department of Dentistry, Ospedale Papa Giovanni XXIII, Bergamo, Italy

23. Department of Oral and Maxillofacial Surgery, Rigshospitalet, Copenhagen University Hospital, Copenhagen, Denmark

24. First Department of Medicine, Semmelweis University Medical School, Budapest, Hungary

25. Department of Oral Surgery, King's College Hospital, London, UK

26. Department of Maxillofacial Surgery, University Hospital of Sassari, Italy

27. Department of Dentistry, San Francesco Hospital, Nuoro, Italy

28. University Hospital Aintree, Liverpool, UK

29. Institute of Translational Medicine, University of Liverpool, Liverpool, UK

30. Department of Systems Biology, Columbia University, New York, US

31. GENVABO Consortium: a list of contributing members is provided below *

32. University College London Hospitals Biomedical Research Centre, London UK

33.

Corresponding author email id: s.fedele@ucl.ac.uk

* Collaborating investigators and sites of GENVABO Consortium (in alphabetical order by site):

Jose L López-Cedrún (Complexo Hospitalario Universitario da Coruña, Spain); Sanne Madsen (Copenhagen University Hospital, Denmark); Gennaro Sadile, Stefania Leuci (Federico II University, Italy); Cristina Mirelli, Giulio Conti, Rita Maiavacca (Fondazione IRCCS Cà Granda, Ospedale Maggiore Policlinico, Italy); Masahiro Urade, Hiromitsu Kishimoto, Shin Okui, Yusuke Zushi, Michiyo Yamamura, Kyohei Yoshikawa (Hyogo College of Medicine, Japan); Giovanna Mansueto (IRCCS, Referral Cancer Center of Basilicata, Italy); Patrik KE Magnusson (Karolinska

This article is protected by copyright. All rights reserved. 
Institutet, Sweden); Shina Popat (King's College Hospital, UK); Barbara Kirnbauer, Barbara Obermayer-Pietsch, Norbert Jakse (Medical University Graz, Austria); Umberto Mariani, Valeria Martini (Ospedale Papa Giovanni XXIII, Italy); Antonella Fasciolo, Iolanda De Martino, Manuela Alessio (Ospedale SS Antonio e Biagio e C Arrigo, Italy); Jacinto F Sanromán (Policlinico Vigo SA, Spain); Sara Grammatico (Sapienza University, Italy); Giovanni Siniscalchi, Angelo Itro (Second University of Naples, Italy); Bernadett Balla, Janos P. Kosa, Mihaly Vaszilko (Semmelweis University Medical School, Hungary); John Lo (The University of Hong Kong, Hong Kong); Eleni Besi, Francesco D’Aiuto, Kwee Yong, Nikos Donos, Valeria Mercadante (University College London, UK); Robert Stein, Shirley D’Sa, (University College London Hospital, UK); Elena Varoni (Università degli Studi di Milano, Italy); Damiano Soma, Silvio Meloni (University Hospital of Sassari, Italy); Jane Evely, Anita Hanson (University of Liverpool, UK); Francesco Giancola, Olga Di Fede, Vera Panzarella (University of Palermo, Italy); Giordana Bettini (University of Padua, Italy); Elisabetta Merigo, Giovanni Mergoni, Paolo Vescovi (University of Parma, Italy); Sergio Gandolfo, Roberto Marino, Mattia Berrone (University of Turin, Italy); Alessio Gambino, Elisa Menegatti, Roberto Broccoletti (University of Turin, CIR Dental School, Italy); Elena Hens, Leticia Bagan (University of Valencia, Spain).

Running title: Time to onset of bisphosphonate-related osteonecrosis of the jaws

Correspondence to: Dr Stefano Fedele, University College London/University College London Hospital Eastman Dental Institute and Hospital, 256 Gray's Inn Road, WC1X 8LD London UK.

\section{ABSTRACT}

Objectives: Osteonecrosis of the jaw (ONJ) is a potentially severe adverse effect of bisphosphonates (BP). Although the risk of ONJ increases with increasing duration of BP treatment, there are currently no reliable estimates of the ONJ time to onset (TTO). The objective of this study was to estimate the TTO and associated risk factors in BP-treated patients.

Subjects and methods: Retrospective analysis of data from 22 secondary care centres in 7 countries relevant to 349 patients who developed BP-related ONJ between 2004 and 2012.

Results: The median (95\%CI) TTO was 6.0 years in patients treated with alendronate $(n=88)$ and 2.2 years in those treated with zoledronate $(n=218)$. Multivariable Cox regression showed that dentoalveolar surgery was inversely associated, and the use of antiangiogenics directly associated, with the TTO in cancer patients treated with zoledronate.

This article is protected by copyright. All rights reserved. 
Conclusions: The incidence of ONJ increases with the duration of BP therapy, with notable differences observed with respect to BP type and potency, route of administration and underlying disease. When data are stratified by BP type, a time of 6.0 and 2.2 years of oral alendronate of intravenous zoledronate therapy, respectively, is required for $50 \%$ of patients to develop ONJ. After stratification by disease, a time of 5.3 and 2.2 years of BP therapy is required for $50 \%$ of patients with osteoporosis and cancer, respectively, to develop ONJ. These findings have significant implications for the design of future clinical studies and the development of risk reduction strategies aimed at either assessing or modulating the risk of ONJ associated with BP.

\section{INTRODUCTION}

Osteonecrosis of the jaw (ON)) is a serious adverse effect of therapy with bisphosphonates (BP) and other anti-resorptive agents (Ruggiero et al, 2009; Sivolella et al, 2013). Affected individuals often present with areas of necrotic ischemic jawbone exposed through fenestration of the oral mucosa or facial skin (Filleul et al, 2010), while approximately one in four patients present with necrotic jawbone covered by intact mucosa (non-exposed variant) (Fedele et al, 2010). Other manifestations include pain, secondary infection, tooth loss, fistula formation, pathological fractures, sinusitis, and oro-antral communication (Filleul et al, 2010). It has been estimated that $\mathrm{ONJ}$ develops in up to $7 \%$ of cancer patients using intravenous BP and in approximately $0.12 \%$ of osteoporosis patients taking oral BP (Kühl et al, 2012).

The pathogenic mechanisms of, and the risk factors for, $\mathrm{ONJ}$ are still controversial (Landesberg et al, 2011). For instance, several studies have reported that the risk of ONJ is greater in patients who have undergone surgical procedures to the jaw bones (e.g. dental extraction)(Campisi et al, 2014). However, it is also known that many ONJ cases, possibly up to 30-40\%, are not triggered by surgical interventions (Filleul et al, 2010). Less controversial is the association between the incidence of $\mathrm{ONJ}$ and the cumulative dose and duration of BP treatment (Thumbigere-Math et al, 2012; Fleisher et al, 2013), in keeping with type C adverse drug reactions (Edwards and Aronson, 2000). Indeed, the incidence of ONJ is low during the first few years of BP treatment and increases substantially thereafter (Barasch et al, 2011). However, it is important to note that there is a great variability and inconsistency in the time to ONJ (TTO) reported in the literature, possibly because of different study designs and diagnostic criteria, and generally low sample sizes (Bamias et al, 2005; Pozzi et al, 2007; Mavrokokki et al, 2007; Boonyapakorn et al, 2008; Vahtsevanos et al, 2009; Saia et al, 2010; Hasegawa et al, 2012; Watters et al, 2013).

A precise estimate of TTO is important for the design of clinical trials. Trials where follow-up is too short would in fact miss most incident cases and provide flawed estimates of ONJ incidence and its risk factors. A precise estimate of TTO is also important for developing risk reduction strategies (e.g. BP dosage reduction or cessation) and surveillance programs (Fedele et al, 2009). In order to obtain a more precise estimate of TTO, we have studied the clinical data collected in GENVABO, a multicentre cross-sectional study aimed at identifying genetic variants predisposing to BP-related ONJ.

This article is protected by copyright. All rights reserved. 


\section{METHODS}

\section{Study design}

We performed a retrospective secondary analysis of data belonging to a cohort of ONJ patients enrolled into the GENVABO (GENetic VAriants as Biomarkers of jaw Osteonecrosis associated with bisphosphonates) study, a genome-wide association study with the primary aim of identifying genetic variants that predispose to ONJ. The present report follows the STROBE recommendations (von Elm et al, 2008).

\section{Setting and inclusion criteria}

GENVABO study was designed by Investigators at the University College London and included a total of twenty-two international clinical centres with an interest in the diagnosis and management of ONJ. The Ethics Committees of the coordinating centre (Central London REC 4, reference 08/H0715/69) and participating sites approved the study and all patients gave their written informed consent to participate. Patients referred to the participating centres between January 2004 and June 2012 were eligible for GENVABO if they had: 1) ONJ diagnosed as per AAOMS criteria (Ruggiero et al, 2009) (Ruggiero et al, 2014); and 2) non-exposed ONJ defined as reported by Fedele et al. and other authors (Junquera and Gallego, 2008; Fedele et al, 2010; Patel et al, 2011). ONJ was diagnosed and adjudicated in all cases by local multidisciplinary teams of specialists in Oral Medicine, Oral and Maxillofacial Surgery, Oncology, Haematology, Rheumatology and Radiology.

\section{Data collection}

Clinical charts of consecutive ONJ patients recruited into the GENVABO study between January 2004 and June 2012 were reviewed and the data of interest were collected between October 2008 and June 2012. Such data were extracted by local investigators and entered into a standardized case report form. All data were inputted into a definitive database using a double entry process performed by two different investigators. The data extracted for the present analysis included: 1) age, gender and race; 2) details of BP therapy including BP type, date of start and length of therapy, and indication for BP use; 3 ) details of ONJ including date of diagnosis, site and type; 4) dental history including history of dentoalveolar surgery and use of dentures preceding ONJ diagnosis; 5) medical history including type 2 diabetes mellitus (T2DM) and use of corticosteroids and antiangiogenics; 6) smoking history; and 7) recruiting centre and relevant country. For patients who had been treated with more than one type of BP, the BP used for the longest time was used for the present analysis. The dataset was reviewed by a central study panel and underwent data cleaning and verification according to standard procedures. Stata 14.1 (Stata Corp., College Station, TX, US) programs were written to ensure the reproducibility of data management and data cleaning.

This article is protected by copyright. All rights reserved. 


\section{Study objectives}

The main aim of GENVABO is to identify genetic variants associated with the risk of developing ONJ. The primary objective of the present secondary analysis was to estimate TTO in patients with BP-related ONJ. TTO was defined as the number of years elapsed between the initiation of $\mathrm{BP}$ therapy and the diagnosis of $\mathrm{ONJ}$ as outlined above. We did not attempt to differentiate the time to diagnosis from the time to development/onset, as the early symptoms of ONJ can be non-specific and they are difficult to assess retrospectively. We also calculated the cumulative incidence of $\mathrm{ONJ}$ and evaluated the association of TTO with potential risk factors (ThumbigereMath et al, 2012; Hasegawa et al, 2012; Fleisher et al, 2013).

\section{Statistical analysis}

The point estimates and the 95\% confidence interval of TTO were calculated using the KaplanMeier estimator (Hosmer et al, 2011). Kaplan-Meier curves for TTO were stratified by disease (metastatic breast cancer $v s$. multiple myeloma vs. metastatic prostate cancer vs. other cancers $v s$. osteoporosis), cancer (yes vs. no) and BP (alendronate vs. ibandronate vs. pamidronate $v s$. zoledronate). Multivariable Cox regression was used to test whether TTO was associated with gender (discrete, male vs. female), age (continuous, decade), dentoalveolar surgery (discrete, yes vs. no), T2DM (discrete, yes vs. no), use of steroids (discrete, yes vs. no) and use of antiangiogenics (discrete, yes vs. no) in cancer patients (Model 1) and in non-cancer patients (Model 2) (Hosmer et al, 2011). Cluster confidence intervals were calculated using the study country as cluster. The proportional hazard assumption made by Cox regression was checked using Schoenfeld residuals (Hosmer et al, 2011). Multivariable fractional polynomials were used to test whether the multivariable relationship of TTO with age was linear (Royston and Sauerbrei, 2008). Statistical analysis was performed using Stata version 14.1.

\section{RESULTS}

\section{Details of the cohort}

Clinical notes of 384 consecutive patients with BP-related ONJ recruited into GENVABO study were available for analysis. Missing or conflicting data were identified for 35 (9\%) patients, who were excluded from further analysis. The majority of the 349 analysed patients (Table 1) were of Caucasian origin (93\%); $85 \%$ were aged $\geq 60$ years and $71 \%$ were females. The majority $(n=$ 318; 91\%) of the participants had exposed ONJ. The most common indications for ONJ treatment were osteoporosis (OP, 32\%), multiple myeloma (MM, 27\%), metastatic breast cancer (MBC, 24\%), and metastatic prostate cancer (MPC, 10\%). Zoledronate (ZOL, 63\%) and alendronate (ALE, 25\%) were the two most commonly employed BP, followed by pamidronate (PAM, 5\%), ibandronate (IBA, 4\%) and risedronate (RIS, 3\%). Concomitant corticosteroids and antiangiogenics (bevacizumab, sunitinib, thalidomide, lenalidomide, bortezomib) were used in $22 \%$ and $14 \%$ of patients respectively. Dentoalveolar surgery preceding ONJ development was reported by $53 \%$ of patients, tobacco smoking by $21 \%$ and T2DM by $10 \%$.

This article is protected by copyright. All rights reserved. 


\section{Time to ONJ onset}

In the whole cohort $(n=349)$, the $50^{\text {th }}(95 \% \mathrm{CI})$ percentile of TTO was 3.2 (2.8 to 3.7$)$ years, the $25^{\text {th }}$ percentile 1.7 (1.4 to 1.9 ) years, and the $75^{\text {th }}$ percentile 5.8 (5.2 to 6.2 ) years. The minimum and maximum TTO were 0.1 and 19.9 years, respectively.

Table 1 shows the median TTO after stratification on several variables including disease and BP type. In brief, when stratified by BP type, the median ( $50^{\text {th }}$ percentile) TTO was 6.0 (5.3 to 6.4) years for ALE and 2.2 (2.1 to 2.6) for ZOL. When stratified by disease, the median TTO was 5.3 (4.4 to 6.1) years for OP, 3.1 (2.2 to 3.6) for MBC and 2.3 (2.1 to 3.0) for MM. The median TTO was 2.2 (2.1 to 2.8 ) years for all cancer patients $(n=237)$.

Figures 1A, 1B and 1C present the Kaplan-Meier plots of the cumulative incidence of ONJ in patients stratified by disease, cancer and BP type. Figure 1A shows that ONJ developed faster in patients with cancer than in those with osteoporosis. Among patients with different cancer types, development was most rapid in those with MPC, followed by OC, MM and MBC (Figure 1C). Figure 1B shows that ONJ developed faster in patients treated with ZOL, RIS and IBA than in those treated with PAM and ALE.

Table 2 shows the multivariable Cox regression models used to evaluate the association between TTO and potential predictors in cancer and non-cancer patients. Model 1 refers to cancer patients taking ZOL (212 out of 237 patients with cancer, 89\%) while Model 2 refers to non-cancer patients taking ALE (84 out of 112 patients, 75\%). A history of dentoalveolar surgery was inversely associated (hazard ratio, $\mathrm{HR}=0.71,95 \% \mathrm{CI} 0.56$ to 0.91 ) and the use of antiangiogenics (HR $=1.10,95 \% \mathrm{CI} 1.01$ to 1.19$)$ directly associated with TTO in the subgroup of cancer patients taking ZOL (Model 1).

\section{DISCUSSION}

Cumulative dosage and duration of anti-resorptive therapy are two of the most consistently reported risk factors for ONJ development (Thumbigere-Math et al, 2012; Fleisher et al, 2013) and there remains little doubt that $\mathrm{ONJ}$ is a time- and dose-related adverse effect. However, data regarding TTO are controversial as they vary significantly among studies (Palaska et al, 2009). Fleisher et al. (Fleisher et al, 2013) reported a median time of 3 and 5 years for ONJ to develop in individuals using intravenous and oral BP respectively, whereas a 2009 review reported a mean time of 1.8 and 4.6 years after ZOL and ALE therapy, respectively (Palaska et al, 2009). Other authors reported that ONJ developed after only 4 months of ZOL therapy, which they suggest was possibly triggered by invasive dental surgical procedures (Saussez et al, 2009). Moreover, a recent study of 191 ONJ cases recruited in a primary care setting (dental practicebased research network) reported a 10-fold increase in risk of $\mathrm{ONJ}$ associated with $<2$ years of BP therapy, which increased to 40 -fold among individuals treated with BP for more than 2 years (Barasch et al, 2011).

Such inconsistency in the data on TTO has negative clinical consequences as it can hinder the delivery of potential risk-reduction strategies such as prophylactic dental measures and BP

This article is protected by copyright. All rights reserved. 
dosage reduction or discontinuation. It can also affect the delivery of clinical surveillance programs (Kyle et al, 2007; Kyrgidis et al, 2013) and cause confusion in the interpretation of clinical studies. For example, clinical trials with a short observation time, e.g. shorter than the median TTO, would miss a significant number of incident cases and therefore provide flawed estimates of $\mathrm{ONJ}$ incidence and its risk factors. The most likely reasons accounting for the inconsistency and variability of current TTO estimates are different study designs, generally low sample sizes, ambiguous definitions of TTO, short follow-up times, and diagnostic criteria limited to exposed ONJ (Palaska et al, 2009). The present study was undertaken with the aim of overcoming these limitations. A significant strength of our study is the large sample size $(n=$ 349), making it the largest study performed so far to investigate TTO. Another strength of our study is the use of a strict definition of TTO as the time elapsed between the commencement of BP therapy and ONJ diagnosis, as determined by a multidisciplinary team. Previous studies have interchangeably used TTO as per clinicians' diagnosis and TTO as based on symptoms reported by patients (Bamias et al, 2005; Pozzi et al, 2007; Mavrokokki et al, 2007; Boonyapakorn et al, 2008; Vahtsevanos et al, 2009; Saia et al, 2010; Hasegawa et al, 2012; Watters et al, 2013) and other studies have used unclear diagnostic criteria (Palaska et al, 2009). On the contrary, our study defined TTO precisely and consistently among centres and avoided the bias associated with the mixing of the diagnoses made by physicians and those made by patients (Lazarovici et $a l, 2009)$. The use of strict diagnostic criteria in multicentre cross-sectional cohort studies reduces the risk of selection bias (Hudson et al, 2005).

We estimated a median TTO of 3.2 years in the whole cohort. When stratified by BP type, the median TTO was 6.0 years for ALE and 2.2 for ZOL. The corresponding figures were 2.1 years for IBA, 2.4 years for RIS and 6.2 years for PAM. With respect to the cumulative incidence (numbers and percentages) of individuals being diagnosed with $\mathrm{ONJ}$ at different time points after commencement of BP therapy (Figure 1), our analysis shows that it took 4.1 years for $75 \%$ of ZOL-exposed ONJ patients and 8.5 years for 75\% of ALE-exposed ONJ patients to develop their disease. Also, 50\% of ZOL-exposed and ALE-exposed ONJ individuals developed their disease in 2.2 and 6 years.

Our results are not notably different from those of previous studies, which were mostly singlecentre and had much smaller cohorts. The three largest single-centre studies performed so far include the $60 \mathrm{ZOL}$ ONJ cases reported by Watters et al. (Marx et al, 2007; Watters et al, 2013), the 27 ALE ONJ cases described by Marx et al. (Marx et al, 2007; Watters et al, 2013), and the 31 ZOL- and 16 ALE-related ONJ cases reported by Lazarovici et al. (Lazarovici et al, 2009), which grouped together make up a smaller sample than the one we recruited and studied. These studies reported a median time to onset of 1.75 years for ZOL-related ONJ (Marx et al, 2007; Watters et al, 2013), 5.7 years for ALE-related ONJ (Marx et al, 2007; Watters et al, 2013), and median times of 2 and 5 years the ZOL-related and ALE-related ONJ, respectively (Lazarovici et al, 2009). Our study confirms that ZOL is associated with a shorter TTO with respect to ALE (Bamias et al, 2005; Pozzi et al, 2007; Mavrokokki et al, 2007; Boonyapakorn et al, 2008; Vahtsevanos et al, 2009; Thumbigere-Math et al, 2012; Watters et al, 2013), which is consistent with the greater potency and better bioavailability of intravenous ZOL. However, in the present study, the median TTO was slightly longer than in previous studies for both ZOL and ALE.

This article is protected by copyright. All rights reserved. 
Another strength of this study is the use of multivariable analysis to investigate the joint association of potential risk factors with TTO. Previous studies suggested that a number of risk factors may have an "additive" impact on ONJ pathogenesis, therefore leading to shorter TTO (Bamias et al, 2005; Pozzi et al, 2007; Mavrokokki et al, 2007; Thumbigere-Math et al, 2012; Boonyapakorn et al, 2008; Palaska et al, 2009; Vahtsevanos et al, 2009; Saia et al, 2010; Hasegawa et al, 2012; Fleisher et al, 2013; Watters et al, 2013). These factors included corticosteroid use, smoking, alcohol, T2DM, dental extraction and use of dentures (Bamias et al, 2005; Pozzi et al, 2007; Mavrokokki et al, 2007; Boonyapakorn et al, 2008; Palaska et al, 2009; Vahtsevanos et al, 2009; Saia et al, 2010; Hasegawa et al, 2012; Watters et al, 2013). Our study considered up to 7 risk factors in a multivariable regression model (Harrell et al, 1996) and shows that none of the previously suggested variables is associated with shorter TTO, including dental risk factors (alveolar surgery and use of dentures). In the subgroup of cancer patients taking ZOL, the only factor associated with shorter TTO in the present study was the use of antiangiogenic agents (Table 2). This was not unexpected, as antiangiogenic medications are known to cause ONJ per se. Quite surprisingly, a history of dento-alveolar surgery to the jawbones was associated with a lower hazard for ONJ in the same subgroup of cancer patients taking ZOL. It is not clear why ONJ developed faster in individuals who had not received dento-alveolar surgery. Of note, despite the relatively large number of subjects compared to the predictors 7 for Model 1 and 6 for Model 2), the estimated hazard ratios have wide 95\%CI suggesting that larger samples are needed to estimate these effects more precisely.

A limitation of the present study lies in its retrospective nature. However, provided that the outcome can be thoroughly assessed, retrospective cohort designs offer a number of advantages over prospective cohort designs if the time to outcome is long (Hudson et al, 2005). For example, based on the present study, one can estimate that a hypothetical prospective study observing cancer patients for 2 years after the start of ZOL therapy would identify less than $50 \%$ of incident $\mathrm{ONJ}$ cases. This would not only decrease the confidence with such incidence estimate can be accepted, but also may identify different risk factors from those identifiable with a longer follow-up.

\section{Conclusions}

This is the largest study performed to date to investigate TTO and its risk factors. It has been conducted in a large well-phenotyped multicentre cohort, with a strict definition of the outcomes, clear diagnostic criteria, and the use of multivariable regression modelling. We believe that its findings can be promptly translated into clinical application to inform the design of clinical trials, epidemiological studies and surveillance programs. For example, a follow-up of at least 2.2 and 6.0 years is needed to capture at least $50 \%$ of the incident cases of ONJ in ZOL and ALE users respectively. Corresponding figures of 4.1 and 8.5 years are needed to capture instead 75\% of ZOL-exposed and ALE-exposed ONJ incident cases. Clinicians should expect that

This article is protected by copyright. All rights reserved. 
ZOL-treated cancer patients who also receive anti-angiogenic therapy may develop ONJ earlier than other patients.

\section{Acknowledgements}

The authors declare no conflict of interest.

Funding: SF received funding from the International Serious Adverse Event Consortium (iSAEC) and NIHR UCLH Biomedical Research Centre; SF and PFL received funding from UCL Grand Challenge PhD Scholarship Programme and Inman Charity. MW and PH received funding from the Swedish Research Council (Medicine 521-2011-2440 and 521-2014-3370), Thuréus' foundation for odontology research and the Clinical Research Support (ALF) at Uppsala University. MP is funded via the MRC Centre for Drug Safety Science and is a NIHR Senior Investigator Emeritus.

Role of the Funding source: The funders of the research had no role in the study's design, conduct, analysis and reporting.

Competing Interest Disclosure: All authors declare: no support from any organisation for the submitted work; no financial relationships with any organisations that might have an interest in the submitted work in the previous three years, no other relationships or activities that could appear to have influenced the submitted work.

Authors and non-author contributors: all authors named in the article byline meet the ICMJE requirements for authorship as they contributed to the conception or design of the work; or the acquisition, analysis, or interpretation of data for the work; AND drafted the work or revised it critically for important intellectual content; AND approved the final the version to be published; AND agreed to be accountable for all aspects of the work in ensuring that questions related to the accuracy or integrity of any part of the work are appropriately investigated and resolved. The other non-author contributors listed under the group "GENVABO Consortium" collected data and provided and cared for study patients.

Transparency declaration: The lead author (Stefano Fedele) affirms that the manuscript is an honest, accurate, and transparent account of the study being reported; that no important aspects of the study have been omitted; and that any discrepancies from the study as planned (and, if relevant, registered) have been explained.

This article is protected by copyright. All rights reserved. 


\section{References}

Bamias A, Kastritis E, Bamia C, et al (2005). Osteonecrosis of the jaw in cancer after treatment with bisphosphonates: incidence and risk factors. J Clin Oncol Off J Am Soc Clin Oncol 23: 8580-8587.

Barasch A, Cunha-Cruz J, Curro FA, et al (2011). Risk factors for osteonecrosis of the jaws: a case-control study from the CONDOR dental PBRN.J Dent Res 90: 439-444.

Boonyapakorn T, Schirmer I, Reichart PA, Sturm I, Massenkeil G (2008). Bisphosphonateinduced osteonecrosis of the jaws: prospective study of 80 patients with multiple myeloma and other malignancies. Oral Oncol 44: 857-869.

Campisi G, Fedele S, Fusco V, Pizzo G, Di Fede O, Bedogni A (2014). Epidemiology, clinical manifestations, risk reduction and treatment strategies of jaw osteonecrosis in cancer patients exposed to antiresorptive agents. Future Oncol Lond Engl 10: 257-275.

Edwards IR, Aronson JK (2000). Adverse drug reactions: definitions, diagnosis, and management. Lancet 356: 1255-1259.

Fedele S, Kumar N, Davies R, Fiske J, Greening S, Porter S (2009). Dental management of patients at risk of osteochemonecrosis of the jaws: a critical review. Oral Dis 15: 527-537.

Fedele S, Porter SR, D'Aiuto F, et al (2010). Nonexposed variant of bisphosphonate-associated osteonecrosis of the jaw: a case series. Am J Med 123: 1060-1064.

Filleul 0, Crompot E, Saussez S (2010). Bisphosphonate-induced osteonecrosis of the jaw: a review of 2,400 patient cases. J Cancer Res Clin Oncol 136: 1117-1124.

Fleisher KE, Jolly A, Venkata UDC, Norman RG, Saxena D, Glickman RS (2013). Osteonecrosis of the jaw onset times are based on the route of bisphosphonate therapy.J Oral Maxillofac Surg Off J Am Assoc Oral Maxillofac Surg 71: 513-519.

Harrell FEJ, Lee KL, Mark DB (1996). Multivariable prognostic models: issues in developing models, evaluating assumptions and adequacy, and measuring and reducing errors. Stat Med 15: 361-387.

Hasegawa Y, Kawabe M, Kimura H, Kurita K, Fukuta J, Urade M (2012). Influence of dentures in the initial occurrence site on the prognosis of bisphosphonate-related osteonecrosis of the jaws: a retrospective study. Oral Surg Oral Med Oral Pathol Oral Radiol 114: 318324.

Hosmer DW, Lemeshow S, May S (2011). Applied Survival Analysis: Regression Modeling of Time to Event Data: Second Edition.

Hudson JI, Pope HGJ, Glynn RJ (2005). The cross-sectional cohort study: an underutilized design. Epidemiol Camb Mass 16: 355-359.

Junquera L, Gallego L (2008). Nonexposed bisphosphonate-related osteonecrosis of the jaws: another clinical variant? J Oral Maxillofac Surg Off J Am Assoc Oral Maxillofac Surg 66: 1516-1517.

Kühl S, Walter C, Acham S, Pfeffer R, Lambrecht JT (2012). Bisphosphonate-related osteonecrosis of the jaws--a review. Oral Oncol 48: 938-947.

This article is protected by copyright. All rights reserved. 
Kyle RA, Yee GC, Somerfield MR, et al (2007). American Society of Clinical Oncology 2007 clinical practice guideline update on the role of bisphosphonates in multiple myeloma. $J$ Clin Oncol Off J Am Soc Clin Oncol 25: 2464-2472.

Kyrgidis A, Tzellos T-G, Toulis K, Arora A, Kouvelas D, Triaridis S (2013). An evidence-based review of risk-reductive strategies for osteonecrosis of the jaws among cancer patients. Curr Clin Pharmacol 8: 124-134.

Landesberg R, Woo V, Cremers S, et al (2011). Potential pathophysiological mechanisms in osteonecrosis of the jaw. Ann N Y Acad Sci 1218: 62-79.

Lazarovici TS, Yahalom R, Taicher S, Elad S, Hardan I, Yarom N (2009). Bisphosphonate-related osteonecrosis of the jaws: a single-center study of 101 patients.J Oral Maxillofac Surg Off J Am Assoc Oral Maxillofac Surg 67: 850-855.

Marx RE, Cillo JEJ, Ulloa JJ (2007). Oral bisphosphonate-induced osteonecrosis: risk factors, prediction of risk using serum CTX testing, prevention, and treatment. J Oral Maxillofac Surg Off J Am Assoc Oral Maxillofac Surg 65: 2397-2410.

Mavrokokki T, Cheng A, Stein B, Goss A (2007). Nature and frequency of bisphosphonateassociated osteonecrosis of the jaws in Australia. J Oral Maxillofac Surg Off J Am Assoc Oral Maxillofac Surg 65: 415-423.

Palaska PK, Cartsos V, Zavras AI (2009). Bisphosphonates and time to osteonecrosis development. The oncologist 14: 1154-1166.

Patel V, McLeod NMH, Rogers SN, Brennan PA (2011). Bisphosphonate osteonecrosis of the jaw-a literature review of UK policies versus international policies on bisphosphonates, risk factors and prevention. Br J Oral Maxillofac Surg 49: 251-257.

Pozzi S, Marcheselli R, Sacchi S, et al (2007). Bisphosphonate-associated osteonecrosis of the jaw: a review of 35 cases and an evaluation of its frequency in multiple myeloma patients. Leuk Lymphoma 48: 56-64.

Royston P, Sauerbrei W (2008). Multivariable Model-Building: A Pragmatic Approach to Regression Analysis based on Fractional Polynomials for Modelling Continuous Variables.

Ruggiero SL, Dodson TB, Assael LA, Landesberg R, Marx RE, Mehrotra B (2009). American Association of Oral and Maxillofacial Surgeons Position Paper on BisphosphonateRelated Osteonecrosis of the Jaw - 2009 update. Aust Endod J 35: 119-130.

Ruggiero SL, Dodson TB, Fantasia J, Goodday R, Aghaloo T, Mehrotra B, O'Ryan

F; American Association of Oral and Maxillofacial Surgeons (2014). American Association of Oral and Maxillofacial Surgeons position paper on medication-related osteonecrosis of the jaw--2014 update. J Oral Maxillofac Surg. 72: 1938-56.

Saia G, Blandamura S, Bettini G, et al (2010). Occurrence of bisphosphonate-related osteonecrosis of the jaw after surgical tooth extraction. J Oral Maxillofac Surg Off J Am Assoc Oral Maxillofac Surg 68: 797-804.

Saussez S, Javadian R, Hupin C, et al (2009). Bisphosphonate-related osteonecrosis of the jaw and its associated risk factors: a Belgian case series. The Laryngoscope 119: 323-329.

This article is protected by copyright. All rights reserved. 
Sivolella S, Lumachi F, Stellini E, Favero L (2013). Denosumab and anti-angiogenetic drugrelated osteonecrosis of the jaw: An uncommon but potentially severe disease. Anticancer Res 33: 1793-1798.

Thumbigere-Math V, Tu L, Huckabay S, et al (2012). A retrospective study evaluating frequency and risk factors of osteonecrosis of the jaw in 576 cancer patients receiving intravenous bisphosphonates. Am J Clin Oncol 35: 386-392.

Vahtsevanos K, Kyrgidis A, Verrou E, et al (2009). Longitudinal cohort study of risk factors in cancer patients of bisphosphonate-related osteonecrosis of the jaw. J Clin Oncol Off J Am Soc Clin Oncol 27: 5356-5362.

von Elm E, Altman DG, Egger M, Pocock SJ, Gøtzsche PC, Vandenbroucke JP (2008). The Strengthening the Reporting of Observational Studies in Epidemiology (STROBE) statement: guidelines for reporting observational studies. J Clin Epidemiol 61: 344-349.

Watters AL, Hansen HJ, Williams T, et al (2013). Intravenous bisphosphonate-related osteonecrosis of the jaw: long-term follow-up of 109 patients. Oral Surg Oral Med Oral Pathol Oral Radiol 115: 192-200.

\section{Tables and figures}

Table 1: Details of the study cohort and time to event stratified by potential risk factors.

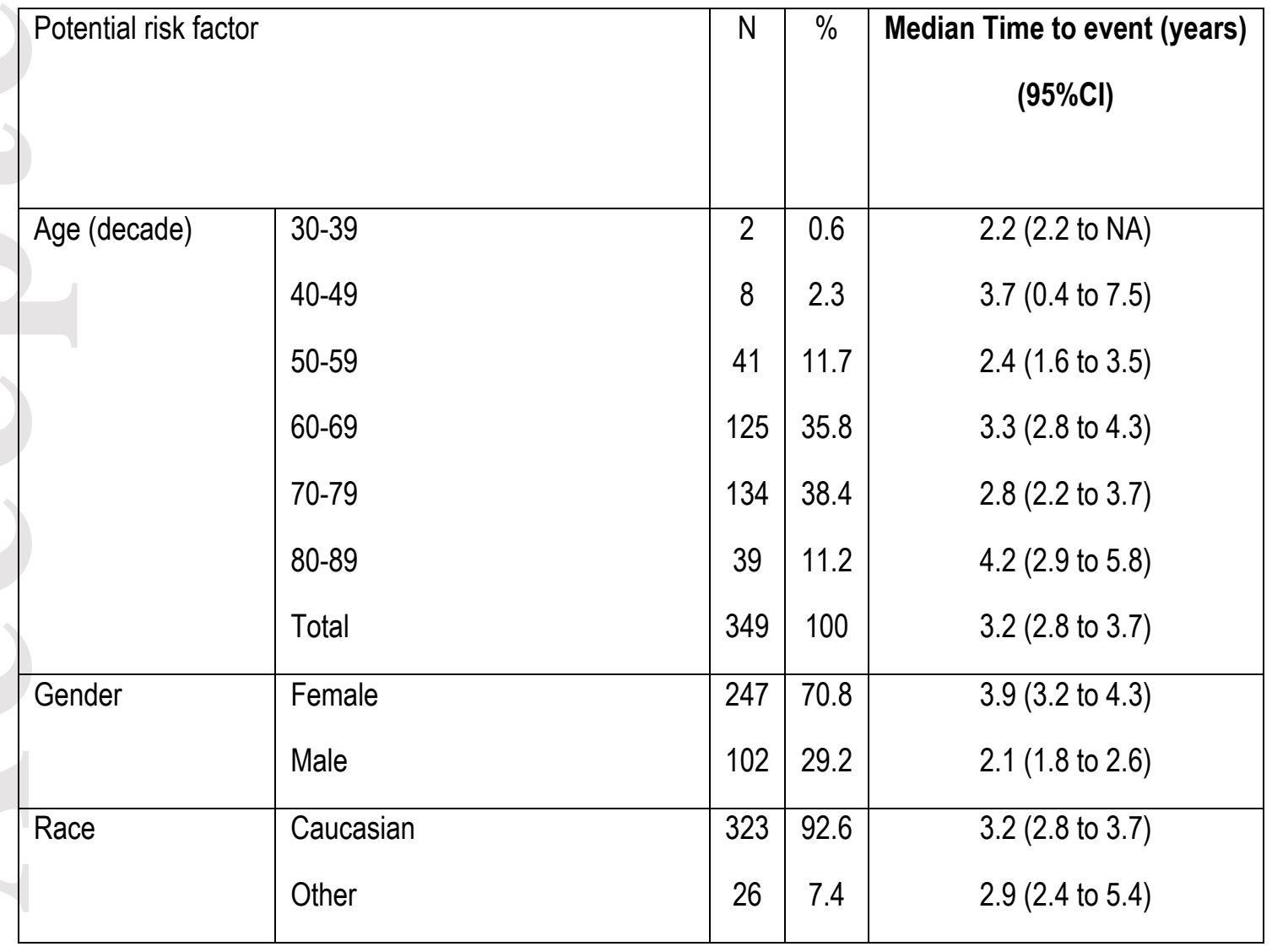

This article is protected by copyright. All rights reserved. 


\begin{tabular}{|c|c|c|c|c|}
\hline Underlying disease & $\begin{array}{l}\text { Metastatic Breast Cancer (MBC) } \\
\text { Multiple Myeloma (MM) } \\
\text { Metastatic Prostate Cancer (MPC) } \\
\text { Other Cancers (OC) } \\
\text { Osteoporosis (OP) }\end{array}$ & $\begin{array}{l}84 \\
93 \\
33 \\
27 \\
112\end{array}$ & $\begin{array}{l}24.1 \\
26.6 \\
9.5 \\
7.7 \\
32.1\end{array}$ & $\begin{array}{l}3.1(2.2 \text { to } 3.6) \\
2.3(2.1 \text { to } 3.0) \\
1.8(1.6 \text { to } 2.1) \\
2.1(1.0 \text { to } 2.8) \\
5.3(4.4 \text { to } 6.1)\end{array}$ \\
\hline BP type & $\begin{array}{l}\text { Alendronate (ALE) } \\
\text { Ibandronate (IBA) } \\
\text { Pamidronate (PAM) } \\
\text { Risedronate (RIS) } \\
\text { Zoledronate (ZOL) }\end{array}$ & $\begin{array}{r}88 \\
15 \\
17 \\
11 \\
218\end{array}$ & $\begin{array}{l}25.2 \\
4.3 \\
4.9 \\
3.2 \\
62.5\end{array}$ & $\begin{array}{l}6.0(5.3 \text { to } 6.4) \\
2.1(0.6 \text { to } 3.2) \\
6.2(4.6 \text { to } 7.2) \\
2.4(0.3 \text { to } 4.7) \\
2.2(2.1 \text { to } 2.6)\end{array}$ \\
\hline Medical History & $\begin{array}{l}\text { Corticosteroids } \\
\text { Antiangiogenics* } \\
\text { Type } 2 \text { diabetes mellitus } \\
\text { Smoking }\end{array}$ & $\begin{array}{l}76 \\
49 \\
34 \\
74\end{array}$ & $\begin{array}{l}21.8 \\
14.0 \\
9.7 \\
21.1\end{array}$ & $\begin{array}{l}3.2 \text { (2.6 to } 4.1) \\
2.3(1.8 \text { to } 3.2) \\
2.9(2.3 \text { to } 4.6) \\
3.4(2.6 \text { to } 4.5)\end{array}$ \\
\hline Dental History & $\begin{array}{l}\text { Dento-alveolar surgery } \\
\text { Denture use }\end{array}$ & $\begin{array}{l}186 \\
73\end{array}$ & $\begin{array}{l}53.3 \\
20.9\end{array}$ & $\begin{array}{l}3.9(3.2 \text { to } 4.5) \\
4.2(2.7 \text { to } 5.3)\end{array}$ \\
\hline ONJ features & $\begin{array}{l}\text { Non-exposed type } \\
\text { Exposed type } \\
\text { Maxilla } \\
\text { Mandible } \\
\text { Both maxilla and mandible }\end{array}$ & $\begin{array}{r}31 \\
318 \\
88 \\
227 \\
34\end{array}$ & $\begin{array}{r}8.9 \\
91.1 \\
25.2 \\
65.0 \\
9.7\end{array}$ & $\begin{array}{l}3.3 \text { (1.7 to } 5.8) \\
3.2 \text { (2.8 to } 3.7) \\
3.3(2.7 \text { to } 3.9) \\
2.9 \text { (2.4 to } 3.6) \\
4.2 \text { (3.0 to } 5.1)\end{array}$ \\
\hline
\end{tabular}

$\mathrm{N}=$ number of patients; $\mathrm{NA}=$ not available; ${ }^{*}$ bevacizumab, sunitinib, thalidomide, lenalidomide, bortezomib

This article is protected by copyright. All rights reserved. 
Table 2: Multivariable Cox regression of factors potentially associated with TTO of ONJ in cancer patients taking ZOL (Model 1) and in non-cancer patients taking ALE (Model 2). Cluster confidence intervals were calculated using the study country as cluster.

\begin{tabular}{|c|c|c|}
\hline & MODEL 1 & MODEL 2 \\
\hline & $\begin{array}{l}\text { Cancer patients } \\
\text { taking ZOL }\end{array}$ & $\begin{array}{l}\text { Non-cancer patients } \\
\text { taking ALE }\end{array}$ \\
\hline Male sex (1= yes; $0=$ no $)$ & $1.25[0.92,1.68]$ & $1.12[0.50,2.53]$ \\
\hline Age/10 (years) & $0.97[0.92,1.03]$ & $1.06[0.83,1.34]$ \\
\hline Dentoalveolar surgery $(1=$ yes; $0=$ no $)$ & $0.71 * *[0.92,1.03]$ & $1.03[0.68,1.23]$ \\
\hline Type 2 Diabetes Mellitus ( $1=$ yes; 0 =no) & $1.00[0.92,1.09]$ & $0.94[0.59,1.51]$ \\
\hline Smoking ( $1=$ yes; $0=$ no) & $1.00[0.74,1.36]$ & $1.05[0.70,1.58]$ \\
\hline Corticosteroids ( $1=$ yes; $0=$ no) & $1.18[0.92,1.50]$ & $1.06[0.90,1.25]$ \\
\hline Antiangiogenics $(1=$ yes; $0=$ no $)$ & $1.10 *[1.01,1.19]$ & -- \\
\hline $\mathrm{N}$ & 212 & 84 \\
\hline
\end{tabular}

Values of hazard rates from multivariable Cox regression with $95 \%$ confidence intervals in brackets

${ }^{*} p<0.05,{ }^{* *} p<0.01$

This article is protected by copyright. All rights reserved. 


\section{Figure Captions}

Figure 1: Kaplan-Meier curves of number and percentage of patients developing ONJ over time. The data are obtained from a retrospective cohort study including only patients who developed ONJ (see text for details). Stratification by [A] underlying disease (cancer vs. non-cancer), [B] bisphosphonate type, and [C] underlying disease (all). MBC: metastatic breast cancer; MPC: metastatic prostate cancer; MM: multiple myeloma; OC: other cancers; OP: osteoporosis; ALE: alendronate; PAM: pamidronate, ZOL: zoledronate; IBA: ibandronate; RIS: risedronate.

A

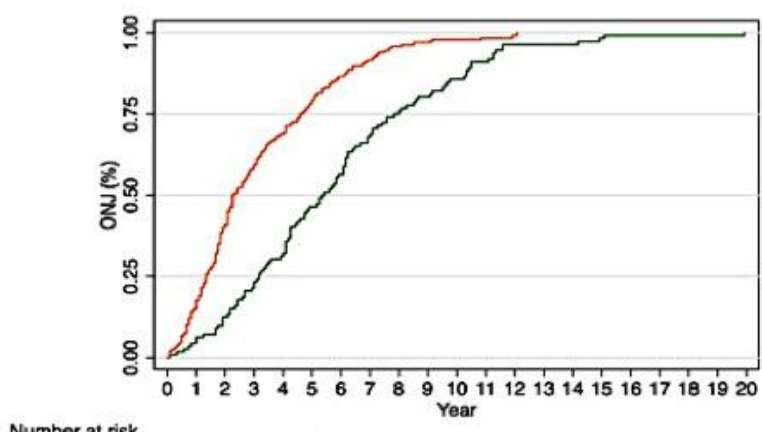

Number at risk

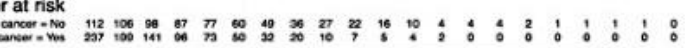

- cancer $=$ No - cancer $=$ Yes

B

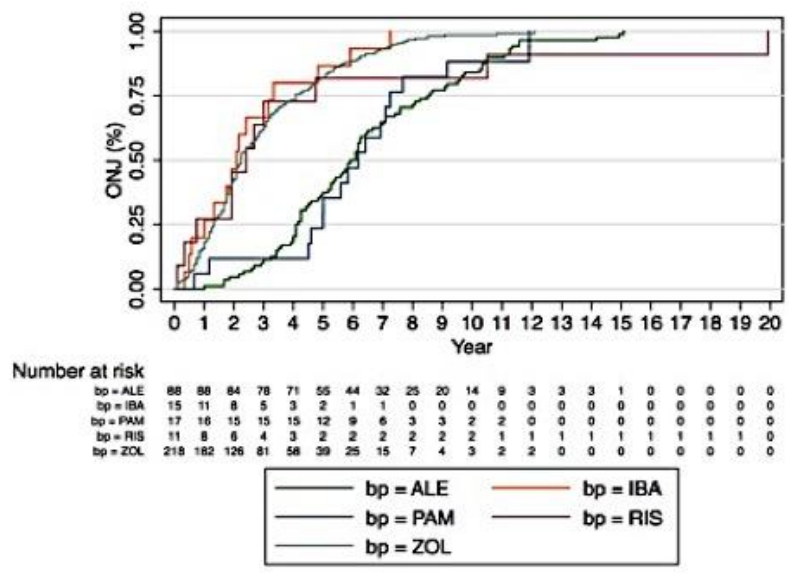

C

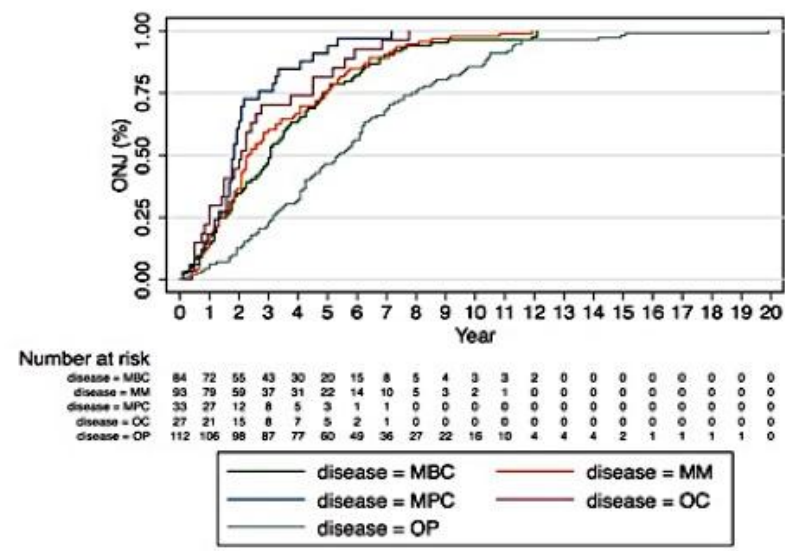

This article is protected by copyright. All rights reserved. 